\title{
Novel Monoclonal Antibodies Provide Evidence for the in situ Existence of a Nonphosphorylated Form of the Largest Neurofilament Subunit
}

\author{
V. M.-Y. Lee, M. J. Carden, and J. Q. Trojanowski \\ Division of Neuropathology, Department of Pathology and Laboratory Medicine, The University of Pennsylvania \\ School of Medicine, Philadelphia, Pennsylvania 19104
}

\begin{abstract}
We have obtained five monoclonal antibodies to the $M, 200,000$ neurofilament component (NF200) after immunization with polypeptides purified from enzymatically dephosphorylated bovine neurofilaments. In immunoblots of untreated neurofilament protein and protein from filaments exposed to phosphatase, these antibodies recognize nonphosphorylated or dephosphorylated, but not phosphorylated, forms of NF200. The epitopes recognized by these new monoclonal antibodies reside in the carboxyterminal domain of the NF200 polypeptide as defined by immunoreaction with limited chymotryptic fragments.

Immunohistochemical studies of bovine cerebellum, spinal cord, trigeminal ganglion, and trigeminal nerve with these new monoclonal antibodies demonstrate immunoreactivity primarily in neuronal perikarya; axons and dendrites are weakly or infrequently immunostained. After enzymatic dephosphorylation of these tissues, a more extensive distribution of immunoreactivity is seen, especially in axons and dendrites. Immunostaining of cultured rat sympathetic neurons is restricted to cell bodies. These data provide evidence for the in situ existence of NF200 epitopes that are not phosphorylated in some classes of neurons or regions of a neuron, but are modified by phosphorylation in other neurons or neuronal domains.

These new monoclonal antibodies are distinctly different from those in a large library (over 100) raised to, and specific for, phosphorylated neurofilament proteins. 'They are novel tools for probing neurofilament distribution, metabolism, structure, and possibly function.
\end{abstract}

Antibodies, particularly monoclonal antibodies, have proved to be powerful probes in defining five immunochemically distinct classes of intermediate filaments (e.g., reviews by Lazarides, 1982; Weber and Geisler, 1984). Neurofilaments are the neuronspecific class of intermediate filaments. In mammals, they are composed of three immunochemically distinct polypeptide subunits, with apparent molecular weights of approximately 200,000 (NF200), 150,000 (NF150) and 68,000 (NF68), as measured by polyacrylamide gel electrophoresis in the presence of SDS-PAGE. Each neurofilament polypeptide is a separate gene product (Czosnek et al., 1980; Lewis and Cowan, 1985), but the three subunits have both unique and shared antigenic determinants

Received May 7, 1985; revised Aug. 23, 1985; accepted Aug. 26, 1985.

This work was supported by N.I.H. Grants NS 18616, CA 36245, Teacher Investigator Development Award NS 00762, and a Postdoctoral Fellowship (M.J.C.) from the Amyotrophic Lateral Sclerosis Foundation of America. Appreciation is expressed to Connie Page and Terri Schuck for excellent technical assistance. Dr. W. W. Schlaepfer provided valuable suggestions and encouragement.

Correspondence should be addressed to Dr. V. M.-Y. Lee, Division of Neuropathology, Dept. of Pathology and Laboratory Medicine, University of Pennsylvania School of Medicine, Johnson Pavilion, Philadelphia, PA 19104.

Copyright (C) 1986 Society for Neuroscience 0270-6474/86/030850-09\$02.00/0 as defined by monoclonal antibodies (e.g., Calvert and Anderton, 1982; Lee et al., 1982b; Sternberger and Sternberger, 1983; Weber and Geisler, 1984). Neurofilaments have been proposed as major determinants of axonal diameter (Hoffman et al., 1984), and have been implicated in the pathogenesis of several neurodegenerative disorders, including Alzheimer's disease (e.g., Dahl et al., 1982; Gambetti et al., 1983; Schlaepfer, 1984), although their precise function remains unknown.

Insights into the function of neurofilaments in normal neurons and in those undergoing degeneration due to disease requires more information about the synthesis, assembly, posttranslational modification, and degradative processing of each neurofilament component. Phosphorylation is an important posttranslational event for modifying, and thereby regulating, protein function in neurons (Nestler and Greengard, 1984). Indeed, it has been recognized for some time that neurofilament polypeptides can bc phosphorylatcd (c.g., Eagles ct al., 1978; Shcckct and Lasek, 1978). More recently, it was shown that freshly isolated mammalian neurofilament proteins contain large amounts of phosphate, particularly in association with the two larger components, NF200 and NF1 50 (e.g., Carden et al., 1985; Geisler et al., 1985; Jones and Williams, 1982; Julien and Mushynski, 1982, 1983). Fragmentation of NF200 and NF1 50 reveals that the major phosphorylation sites are in the carboxy-terminal domains of each polypeptide (Geisler et al., 1985; Julien and Mushynski, 1983). These domains are believed to be peripheral to the $10 \mathrm{~nm}$ diameter backbone of the neurofilament and, at least for NF200, are thought to be involved in forming crossbridge structures between filaments (Chin et al., 1983; Geisler et al., 1985; Hirokawa et al., 1984; Julien and Mushynski, 1983).

The basis for the studies described in this paper is the insightful observation of Sternberger and Sternberger (1983), later confirmed in this laboratory (Carden et al., 1985), that monoclonal antibodies specific for NF200 and/or NF150 recognize epitopes whose immunoreactivities are modified by phosphorylation. We refer to these antigenic determinants as "phosphate-dependent" epitopes. In contrast with our previously described library of monoclonal antibodies to freshly isolated bovine neurofilament proteins (Carden et al., 1985; Lee et al., 1982b, 1984; Trojanowski et al., 1985), we have now used enzymatically dephosphorylated bovine NF200 as an immunogen to produce five new monoclonal antibodies that recognize endogenous dephosphorylated or nonphosphorylated-as well as enzymatically dephosphorylated-bovine NF200 in immunoblots, but not the phosphorylated form of NF200. Immunohistochemistry of normal bovine cerebellum, spinal cord, trigeminal nerve, and trigeminal ganglion using these new monoclonal antibodies yields patterns of immunoreactivity which differ from those obtained with antibodies that recognize epitopes present in phosphorylated NF200. The patterns suggest that, in situ, 


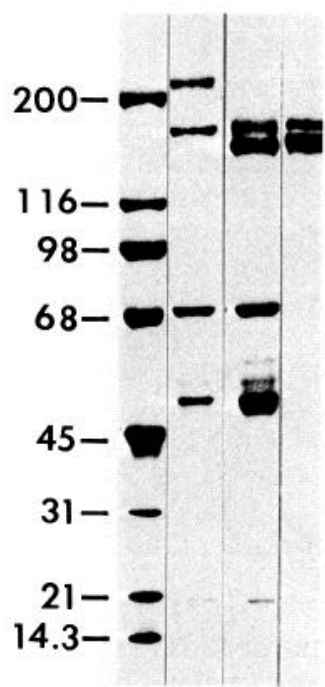

$\begin{array}{llll}1 & 2 & 3 & 4\end{array}$

Figure 1. Coomassie blue-stained $7.5 \%$ polyacrylamide gels of phosphorylated (non-enzyme-treated) and enzymatically dephosphorylated neurofilament proteins. Lane 1 contains the following marker proteins: myosin $(M, 200,000)$, beta-galactosidase $\left(M_{r}, 116,000\right)$, phosphorylase B $(M, 98,000)$, BSA $(M, 68,000)$, ovalbumin $\left(M_{r} 45,000\right)$, carbonic anhydrase $(M, 31,000)$, soybean trypsin inhibitor $(M, 21,000)$, lysozyme $(M, 14,300)$. Lane 2, Phosphorylated (non-enzyme-treated) neurofilament proteins. Lane 3, Enzymatically dephosphorylated neurofilament proteins. Lane 4, Gel-excised, enzymatically dephosphorylated NF150 and NF200 used as immunogens.

NF200 exists in different phosphorylation states within the same neuron and that phosphorylation of NF200 is region-dependent.

\section{Materials and Methods}

\section{Preparation of dephosphorylated neurofilaments and isolation} of dephosphorylated polypeptide components

Neurofilament-rich (over $80 \%$ ) preparations were made from freshly obtained bovine spinal cords that had been enriched for white matter using methods described previously (Carden et al., 1985). These were dephosphorylated with alkaline phosphatase from E. coli (Sigma Chemicals, Type III-N). We used 10 units of enzyme per milligram of neurofilament protein (Carden et al., 1985). Briefly, the phosphatase-neurofilament mixture contained $1 \mathrm{~mm} \mathrm{ZnSO} \mathrm{m}_{4}, 100 \mathrm{mM} \mathrm{NaCl}$, and $50 \mathrm{~mm}$ Tris- $\mathrm{HCl}$ buffer, $\mathrm{pH} 8.0$, and was incubated for $18 \mathrm{hr}$ at $37^{\circ} \mathrm{C}$. Filaments were pelleted from solution by centrifugation at $100,000 \times g$ for $2 \mathrm{hr}$ at $4^{\circ} \mathrm{C}$ to remove excess enzyme. The pellets were dissolved in Laemmli (1970) sample buffer for preparative gel electrophoresis in the presence of SDS (see below).

\section{Chymotryptic digestion of neurofilaments}

Freshly isolated (phosphorylated) or phosphatase-treated (dephosphorylated) neurofilaments were digested with chymotrypsin and centrifuged to separate soluble fragments from sedimentable protein under conditions previously described (Carden et al., 1985).

\section{Extraction of the endogenous nonphosphorylated or dephosphorylated form of NF200 from bovine tissues}

Fresh bovine cerebellar cortex and white matter as well as trigeminal ganglia and trigeminal nerve were dissected under a microscope and homogenized separately in a 10 -fold dilution (wt/vol) of buffer containing $8 \mathrm{M}$ urea, $2 \%$ SDS, $5 \%$ beta-mercaptoethanol, and $0.1 \mathrm{M}$ Tris$\mathrm{HCl}, \mathrm{pH} 6.8$, in a ground glass Duall homogenizer. The homogenates were immediately centrifuged $(100,000 \times \mathrm{g})$ for $30 \mathrm{~min}$, to eliminate myelin and insoluble particles. Without further treatment, the supernatants were subjected to SDS-PAGE ( $7.5 \%$ polyacrylamide) and immunoblotting, as described below.

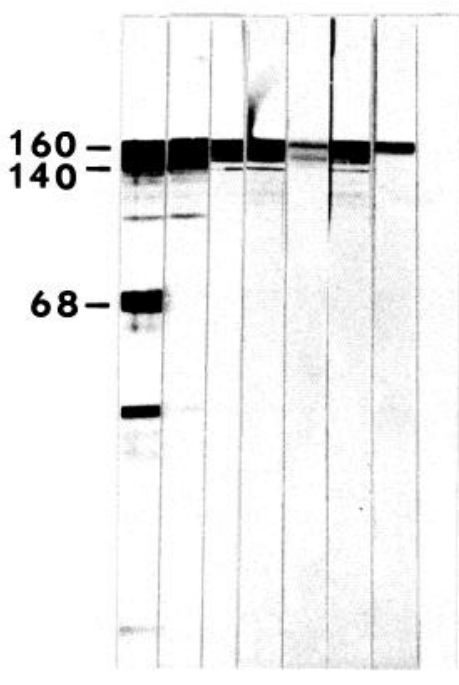

12345678

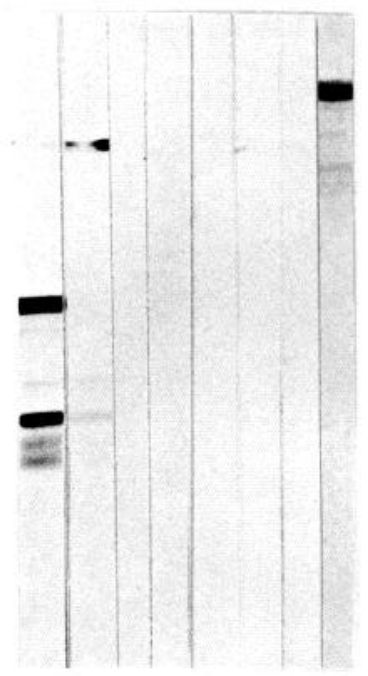

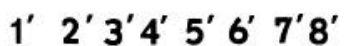

Figure 2. Immunoblots of extracted neurofilament proteins before and after enzymatic dephosphorylation. Strips 1-8 were cut from a nitrocellulose replica of a $7.5 \%$ polyacrylamide gel used to separate phosphatase-treated, i.e., dephosphorylated, bovine neurofilament proteins. Strips $1^{\prime}-8^{\prime}$ contain proteins from freshly isolated, phosphorylated neurofilaments. Strips were incubated with antisera or monoclonal antibodies as follows: 1 and $I^{\prime}$, antiserum to whole, dephosphorylated neurofilaments; 2 and $2^{\prime}$, antiserum to the mixture of pure, dephosphorylated NF150 and NF200; 3 and $3^{\prime}, \mathrm{dP} 1 ; 4$ and $4^{\prime}, \mathrm{dP} 2 ; 5$ and $5^{\prime}, \mathrm{dP} 3 ; 6$ and $6^{\prime}, \mathrm{dP} 4 ; 7$ and $7^{\prime}, \mathrm{dP} 5 ; 8$ and $8^{\prime}, \mathrm{Ta} 50$, a monoclonal antibody from a fusion using phosphorylated NF200 as immunogen. Numbers on the left indicate the apparent $M_{r}$ values of the phosphatase-treated forms of NF200 (160 kDa), NF150 (140 kDa), and NF68 $(M$, unaltered).

\section{Preparation of antisera and monoclonal antibodies}

Antisera and monoclonal antibodies were generated and screened using previously described methods (Lee et al., 1982a, b, 1984). Lewis rat splenocytes were used for the fusion following immunization with purified, dephosphorylated NF150 and NF200. All hybridomas were subcloned at least once. Supernatants were screened by enzyme-linked immunosorbant assay (ELISA) using five different antigens: (1) nondenatured, freshly isolated (i.e., phosphorylated) whole neurofilaments; (2) nondenatured, phosphatase-treated whole filaments; (3) geleluted, phosphorylated NF200 derived from freshly isolated filaments; (4) gel-eluted, phosphorylated NF150, and (5) gel-eluted, dephosphorylated NF200/NF150 derived from phosphatase-treated filaments. Subunit specificity and the domain of the epitope recognized by the antibodies were determined in immunoblots of phosphorylated and phosphatase-treated neurofilaments and chymotryptic fragments thereof. Rat monoclonal antibodies recognizing phosphate-dependent epitopes in bovine NF150 and/or NF200 were also used in this study; they were obtained from earlier fusions produced by use of antigens from freshly isolated, phosphorylated neurofilaments (Carden et al., 1985; Lee et al., 1982b, 1984; Trojanowski et al., 1985). The immune sera from the two rats used in the fusion just described were saved. Another antiserum was obtained following immunization of one rat with whole, phosphatase-treated neurofilaments.

\section{Polyacrylamide gel electrophoresis and immunoblotting}

Polyacrylamide gels $(7.5 \%)$ were used for analytical studies; $6 \%$ polyacrylamide gels were used for preparative electrophoresis leading to gel elution of particular polypeptides. Sample preparation and electrophoresis buffers for SDS gels were according to Laemmli (1970). Nonequilibrium pH-gradient gel electrophoresis (NEPHGE) was performed as described by O'Farrell et al. (1977) using gels containing ampholines (LKB) of pH 3.5-10. Transfer of proteins from SDS gels to nitrocellulose paper was as described by Lee et al. (1984). Nitrocellulose replicas, or blots, were blocked in $1 \%$ BSA, incubated with antisera or hybridoma supernatants, washed, incubated with secondary antibody (peroxidaseconjugated goat anti-rat IgG, Cappell Laboratories), washed again, and 


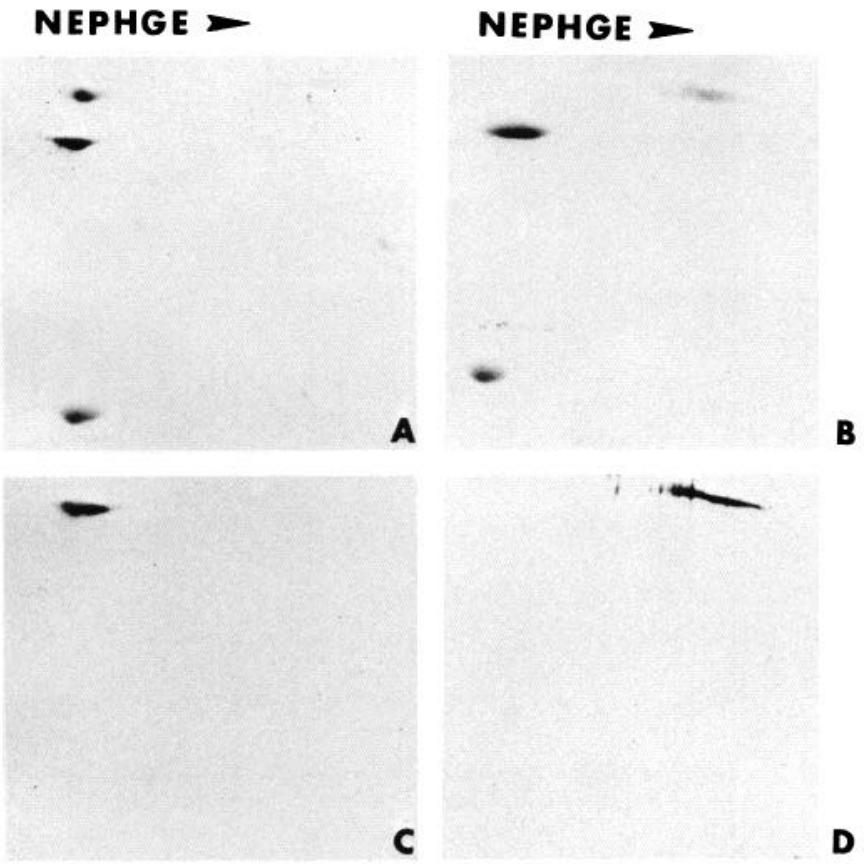

Figure 3. Untreated and enzymatically dephosphorylated NF200 in two-dimensional gels. $A$ and $B$, Coomassie blue-stained two-dimension gels used to separate phosphatase-treated $(B)$ and untreated $(A)$ neurofilaments into their component proteins. The first-dimension NEPHGE gels were run in the direction indicated. $C$ and $D$, Immunoblots of gels indentical to $A$ and $B$, respectively. The blot in $C$ was obtained using the monoclonal antibody Ta50, which recognizes only phosphorylated NF200 (see Figure 2), whereas the blot in $D$ resulted from incubation with dP1, a monoclonal antibody recognizing an epitope in NF200 only when the polypeptide is dephosphorylated.

developed with the chromogen 4-chloro-1-naphthol, as described elsewhere (Lee et al., 1984).

\section{Culture of rat sympathetic neurons}

Dissociated neurons from newborn rat sympathetic ganglia were obtained and cultured as described by Lee and colleagues (Lee, in press; Lee et al., 1981). Long-term cultures were grown on collagen-coated glass coverslips in a medium containing L15 and additives (Lee, in press).

\section{Immunohistochemistry}

Indirect immunofluorescence was performed on frozen sections of fresh bovine cerebellum and spinal cord, as well as on cultured rat sympathetic neurons, after fixation in acetone at $-20^{\circ} \mathrm{C}$ for $7 \mathrm{~min}$. Hybridoma supernatants were applied to tissues for $1 \mathrm{hr}$ at $20^{\circ} \mathrm{C}$, followed by rhodamine-conjugated goat anti-rat IgG (Cappell Laboratories) as secondary antibody, using methods described previously (Lee et al., 1982a, 1984).

Peroxidase anti-peroxidase (PAP) immunohistochemistry was performed on $40-\mu \mathrm{m}$-thick vibratome sections and $6-\mu \mathrm{m}$-thick paraffin sections of bovine cerebellum, spinal cord, trigeminal nerve, and trigeminal ganglion fixed in Bouin's medium (Lee et al., 1982b; Trojanowski et al., 1985). In order to determine whether monoclonal antibodies recognized phosphate-dependent epitopes in situ, vibratome and paraffin sections of these tissues were also treated with phosphatase before immunohistochemistry. Each was incubated at $37^{\circ} \mathrm{C}$ with $300 \mu \mathrm{l}$ of alkaline phosphatase (Sigma Type III-N from E. coli) dissolved (4 to 8 units/ml) in $1 \mathrm{~mm} \mathrm{ZnSO}$, $100 \mathrm{~mm} \mathrm{NaCl}$, and $50 \mathrm{~mm}$ Tris- $\mathrm{HCl}$, pH 8.0. Dephosphorylation was terminated after 2.5 to $3 \mathrm{hr}$ by three washes in buffer. Controls included tissue sections incubated with buffer alone, with supernatant from unfused mouse myeloma cells (as a negative antibody control), or with an anti-glial filament monoclonal antibody (2.2B10) described by Lee et al. (1984).

\section{Results}

\section{Dephosphorylation of neurofilaments}

Figure 1 shows a gel containing samples of freshly isolated bovine neurofilament proteins (lane 2), dephosphorylated neurofilaments resulting from treatment with phosphatase (lane 3), and purified, dephosphorylated NF200 and NF150 polypeptides (lane 4) as used for immunization to produce antibodies. Phosphatase treatment results in removal of more than $90 \%$ of the phosphate from bovine NF200 and NF150 (Carden et al., 1985), and this causes a change in their electrophoretic mobilities in the presence of SDS (compare lanes 2 and 3, Fig. 1). Because dephosphorylated NF200 and NF150 migrate so close together in SDS gels (lane 3), we eluted both bands from preparative gels in one pooled fraction (lane 4, Fig. 1). This mixture was used for immunization of rats.

\section{Antibodies to dephosphorylated neurofilament proteins}

Immunization with the dephosphorylated NF200/NF150 mixture yielded five monoclonal antibodies. The five monoclonal antibodies (named dP1-5) were identified and selected for further characterization on the basis of the ELISA data, which demonstrated that the antibodies were specific for dephosphorylated NF200/NF150, but not for the phosphorylated forms of either polypeptide as purified from freshly isolated neurofilaments. The subunit specificity of each monoclonal antibody (dP1-5) was determined in immunoblots of phosphorylated and enzymatically dephosphorylated neurofilaments (Fig. 2).

The nitrocellulose strips numbered 1 through 8 on the left side in Figure 2 contain enzymatically dephosphorylated neurofilament proteins. The monoclonal antibodies $\mathrm{dP1}-5$ (strips $3-7$, respectively) show strong immunoreactivity to dephosphorylated NF200 (migrating with an apparent $M_{r}$ of approximately 160,000 ), but fail to recognize the phosphorylated form of NF200 from freshly isolated filaments (strips $3^{\prime}-7^{\prime}$, respectively). A monoclonal antibody (Ta50) from a previous fusion (Lee et al., 1982b) made with NF200 from freshly isolated bovine neurofilaments as immunogen (strips 8 and $8^{\prime}$, Fig. 2) recognizes phosphorylated NF200, but not the dephosphorylated form. The specificity of Ta 50 for a phosphate-dependent epitope in NF200 has been documented (Carden et al., 1985), as have other monoclonal antibodies that recognize phosphorylated NF200 more strongly than the dephosphorylated form.

Figure 2 also shows the specificities of antisera raised to the enzymatically dephosphorylated NF150/200 mixture (strips 2 and $2^{\prime}$ ) or to whole, enzymatically dephosphorylated filament preparations (strips 1 and $1^{\prime}$ ). The antiserum to NF150/200 recognizes both polypeptides in their dephosphorylated form (strip 2), but only NF150 in phosphorylated filament preparations (strip $2^{\prime}$ ). The antiserum to whole, dephosphorylated filaments recognizes NF68 and contaminating glial fibrillary acidic protein equally well, both in freshly isolated filaments (strip 1') and in phosphatase-treated protein (strip 1). However, it recognizes only the dephosphorylated forms of NF200 and NF150.

Figure 3 shows two-dimensional gels produced by NEPHGE separation of freshly isolated (Fig. $3 A$ ) and phosphatase-treated (Fig. $3 B$ ) neurofilament preparations followed by SDS-PAGE electrophoresis. NEPHGE separates according to isoelectric point. Because of their reduced phosphate content, dephosphorylated forms of NF150 and particularly of NF200 migrate to much more basic portions of these gels (Fig. $3 B$ ) than do the phosphorylated proteins (Fig. $3 A$ ). The distribution in the gel of enzymatically dephosphorylated NF150 (Fig. $3 B$ ) is wider than that of the phosphorylated form (Fig. $3 A$ ); and enzymatically dephosphorylated NF200 migrates as an elongated streak spanning several $\mathrm{pH}$ unit positions. This streak is centered at about pH 7.5 (Fig. $3 B$ ), in contrast to the sharp spot centered 


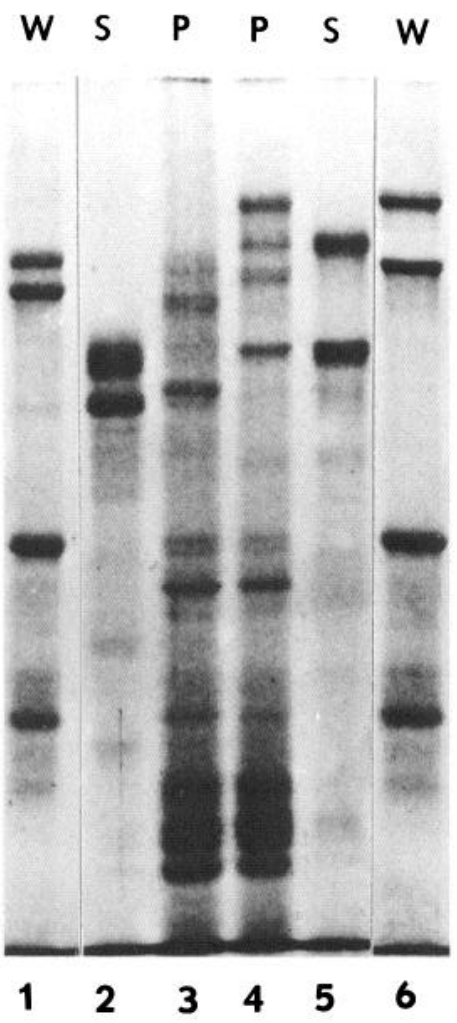

Figure 4. Chymotryptic digests of untreated and enzymatically dephosphorylated neurofilament proteins. Coomassie blue-stained $7.5 \%$ gels of enzymatically dephosphorylated (lanes 1-3) and phosphorylated (lanes 4-6) neurofilament proteins before (lanes 1 and 6) and after (lanes 2-5) digestion with chymotrypsin. $W=$ whole neurofilament preparation prior to digestion with chymotrypsin; $S=$ soluble and $P=$ pelleted fractions after digestion with chymotrypsin.

at approximately pH 5.8 for phosphorylated NF200 (Fig. 3A). Gels identical to Figure $3, A$ and $B$ were immunoblotted with the monoclonal antibodies Ta50 and dP1 (Fig. 3, $C$ and $D$, respectively). Ta50 again recognizes the phosphorylated form of NF200 (Fig. 3C), while dP1 recognizes only the most basic part of the enzymatically dephosphorylated NF200 spot (Fig. $3 D$ ). Antibodies dP2-5 produced results similar to that shown in Figure $3 D$ (data not shown). Two-dimensional NEPHGE/ SDS gels therefore provide further evidence of the fact that dP15 are monoclonal antibodies specific for only the dephosphorylated form of NF200.

Chymotryptic digestion of enzymatically dephosphorylated neurofilaments was used to determine whether the epitopes recognized in NF200 by the monoclonal antibodies dP1-5 are located in peripheral, carboxy-terminal domains of NF200 or in core-associated domains. Figure 4 shows the effect of chymotrypsin upon neurofilaments. Cleaveage of freshly isolated neurofilaments (consisting predominantly of phosphorylated species) releases fragments of apparent $M, 170,000$ and 110,000 that remain in the high-speed supernatant fraction (lane 5, Fig. 4). These are derived from NF200 and NF150, respectively (Carden et al., 1985; Chin et al., 1983; Julien and Mushynski, 1983). Chymotryptic digestion of enzymatically dephosphorylated neurofilaments also produces two major soluble fragments from NF200 and NF150 (lane 2, Fig. 4), although their gel mobilities are much reduced compared with their phosphorylated counterparts (Carden et al., 1985).

Figure 5 shows the results of immunoblotting experiments using the monoclonal antibodies dP1-4 to detect soluble chy-

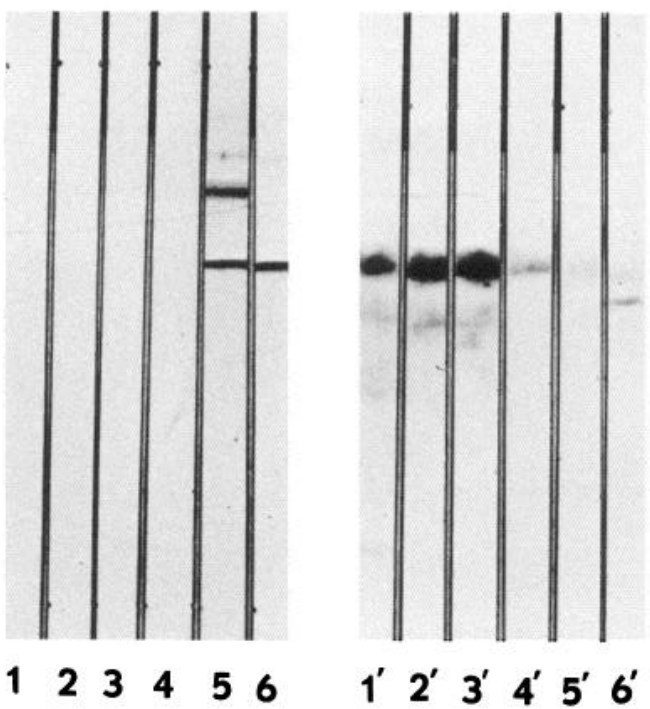

Figure 5. Epitopes recognized by monoclonal antibodies are located in the carboxy-terminal domain of NF200. Strips were cut from nitrocellulose replicas of $7.5 \%$ polyacrylamide gels containing the soluble chymotryptic fragments from phosphorylated (lanes $1-6$ ) or enzymatically dephosphorylated (lanes $\left.1^{\prime}-6^{\prime}\right)$ neurofilaments. The strips were incubated with the following monoclonal antibodies: dP1 ( 1 and $\left.1^{\prime}\right)$; $\mathrm{dP} 2$ ( 2 and $\left.2^{\prime}\right)$; dP3 (3 and $\left.3^{\prime}\right)$; dP5 (4 and $\left.4^{\prime}\right)$; Ta12 (5 and $\left.5^{\prime}\right)$; Oc85 $\left(6\right.$ and $\left.6^{\prime}\right)$. Both dP1-3 and dP5 were raised to enzymatically dephosphorylated neurofilament proteins. Ta 12 and Oc85 were obtained in earlier fusions using phosphorylated antigens.

motryptic fragments of NF150 and NF200 from phosphorylated (strips 1-6) and enzymatically dephosphorylated (strips $1^{\prime}-6^{\prime}$ ) neurofilaments. Each antibody binds the dephosphorylated NF200 chymotryptic fragment, but not the phosphorylated counterpart (compare strips $1^{\prime}-4^{\prime}$ with $\left.1-4\right)$. The monoclonal antibody Ta1 2 (see strips 5 and $5^{\prime}$ ), from our library of monoclonal antibodies against phosphorylated bovine neurofilaments, recognizes a shared epitope that resides in the carboxyterminal domains of NF150 and NF200. The loss of reaction in strip $5^{\prime}$ suggests that both epitopes are phosphate-dependent. Similarly, monoclonal antibody Oc85 (stripes 6 and $6^{\prime}$ ) recognizes a phosphate-dependent epitope that is exclusive to the carboxy-terminal domain of NF150 and thus shows reduced affinity for the dephosphorylated fragment (lane $6^{\prime}$ ).

\section{Immunohistochemical studies with monoclonal antibodies} specific for dephosphorylated NF200

Of the five new monoclonal antibodies (dP1-5), only three (dP1, 3 , and 4 ) consistently stained acetone-fixed nervous tissues by indirect immunofluorescence. The staining patterns of these monoclonal antibodies were compared with those of antibodies specific for the phosphorylated form of NF200 (e.g., Ta50) under the same conditions (Fig. 6). In frozen sections of bovine cerebellum, Purkinje cell bodies were intensely stained by the monoclonal antibodies specific for dephosphorylated NF200, i.e., the $\mathrm{dP}$ series (Fig. 6A). This immunofluorescence pattern is completely different from any observed previously in similar tissue when using antibodies against NF200 isolated by conventional methods, with normal nervous tissues (e.g., Dahl, 1983; Schlaepfer et al., 1981; Trojanowski et al., 1985). Staining with Ta50, which recognizes a phosphate-dependent epitope in NF200, is representative of such previously observed patterns (Fig. $6 B$ ): It fails to stain the perikarya of Purkinje cells (asterisks in $6 B$ ), while intensely staining basket cell axon collaterals and other neurites in the region surrounding Purkinje cells. In con- 

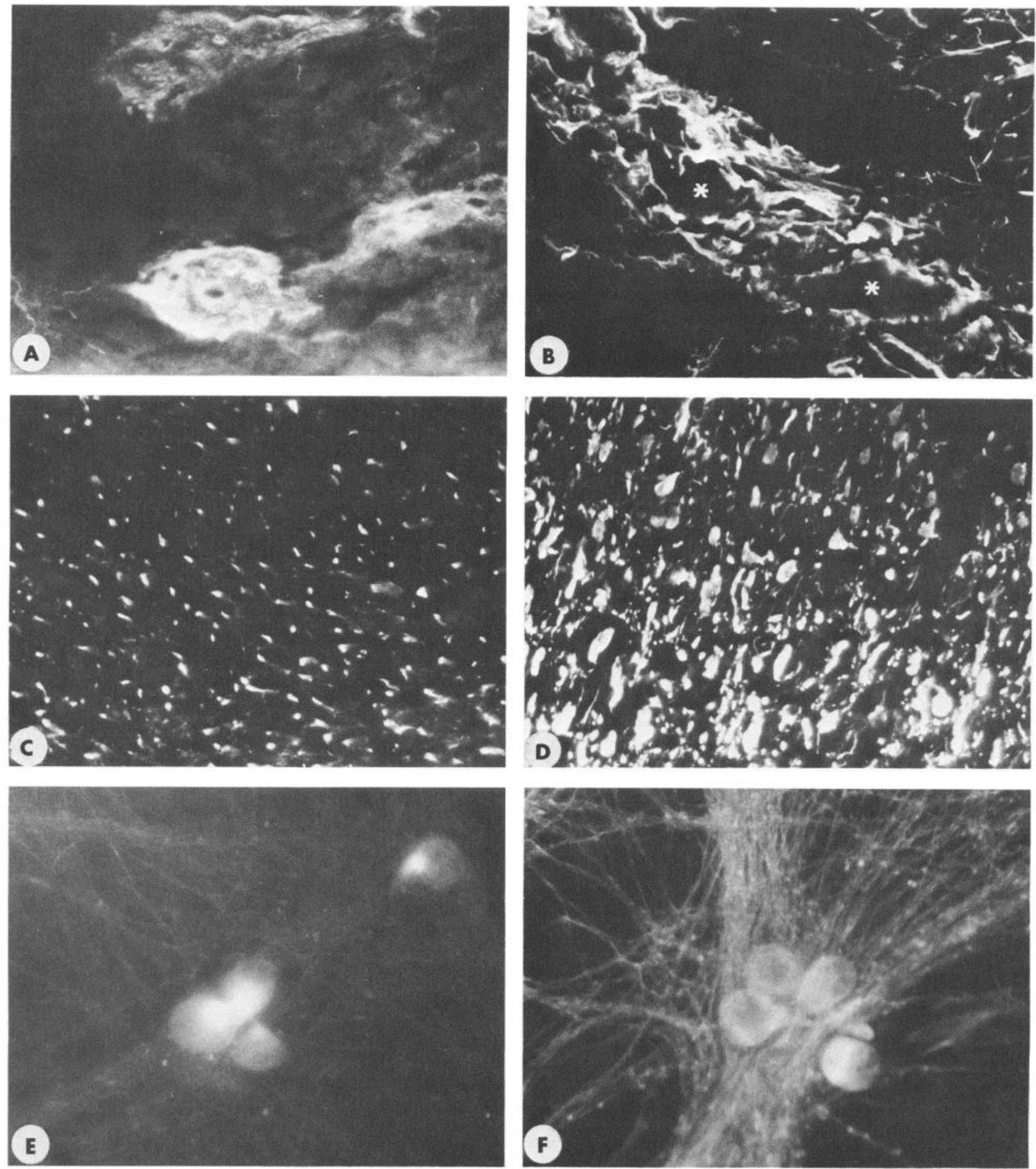

Figure 6. Detection of endogenous forms of nonphosphorylated or dephosphorylated NF200 by immunofluorescence. Indirect immunofluorescence was conducted on acetone-fixed, frozen sections of adult bovine cerebellum $(A$ and $B)$ and spinal cord $(C$ and $D)$ and on 4 week cultures of sympathetic neurons $(E$ and $F)$ also fixed in acetone. Tissues in $A, C$, and $E$ were incubated with $\mathrm{dP} 1$ and those in $B, D$, and $F$ with Ta50. Note differences between staining patterns for these NF200-specific antibodies according to whether the epitope that they recognize is in the phosphorylated $(B, D$, and $F)$ or nonphosphorylated $(A, C$, and $E)$ state. Unstained Purkinje cells in $B$ are identified by asterisks. All magnifications $\times 800$.

trast to these results, Ta50 does stain Purkinje perikarya under different conditions of tissue fixation and embedding using the more sensitive PAP method (see below).

Immunoflourescence of spinal cord sections with these same monoclonal antibodies again reveals differences in immunostaining patterns (compare Fig. 6, $C$ and $D$ ). Ta50 stained many large axons (Fig. $3 D$ ), whereas dP1 stained fewer and smaller axons. Differences in staining between these two antibodies were also observed with immunofluorescence of rat neurons cultured for 4 weeks. Ta50 stained perikarya and processes (Fig. $6 F$ ), but dP1 staining was confined to the cell body (Fig. $6 E$ ).

These immunofluorescence studies, combined with the results of the previous section showing that $\mathrm{dP1}$ and Ta50 recognize epitopes modified by phosphorylation state, suggest that NF200 

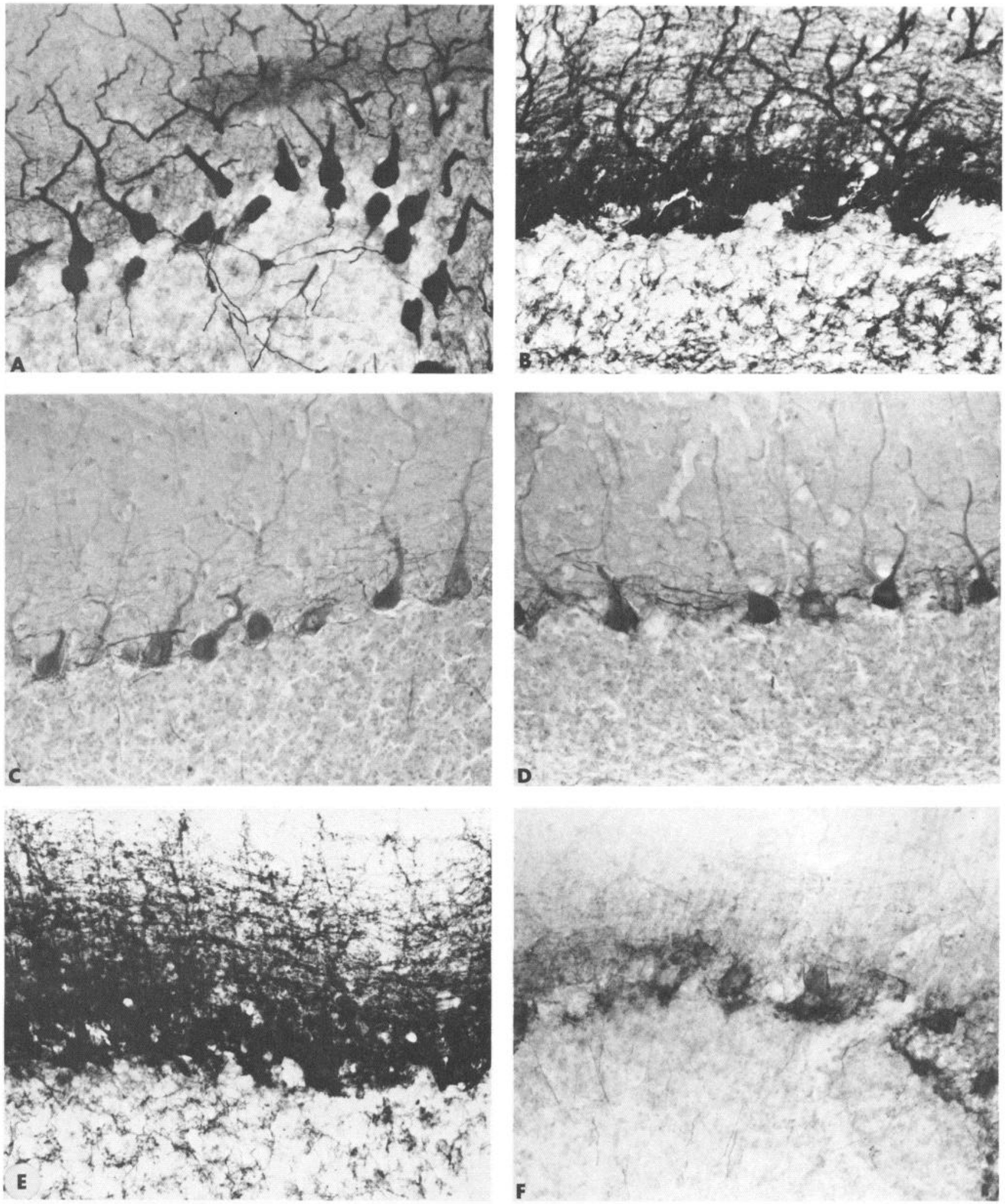

Figure 7. Detection of endogenous forms of nonphosphorylated or dephosphorylated NF200 in bovine cerebellum. Immunoperoxidase studies were conducted on vibratome sections of Bouin's solution-fixed bovine cerebellum incubated with Tris buffer $(A, C$, and $E)$ or with alkaline phosphatase $(B, D$, and $E)$ followed by $\mathrm{dP} 1(A$ and $B)$, Ta56 $(C$ and $D)$, or Ta50 $(E$ and $F)$. Antibody specificities are, for dP1, enzymatically dephosphorylated NF200; for Ta56, phosphate-independent NF200 core epitope; for Ta50, phosphate-dependent NF200 peripheral epitope. All magnifications $\times 200$. 

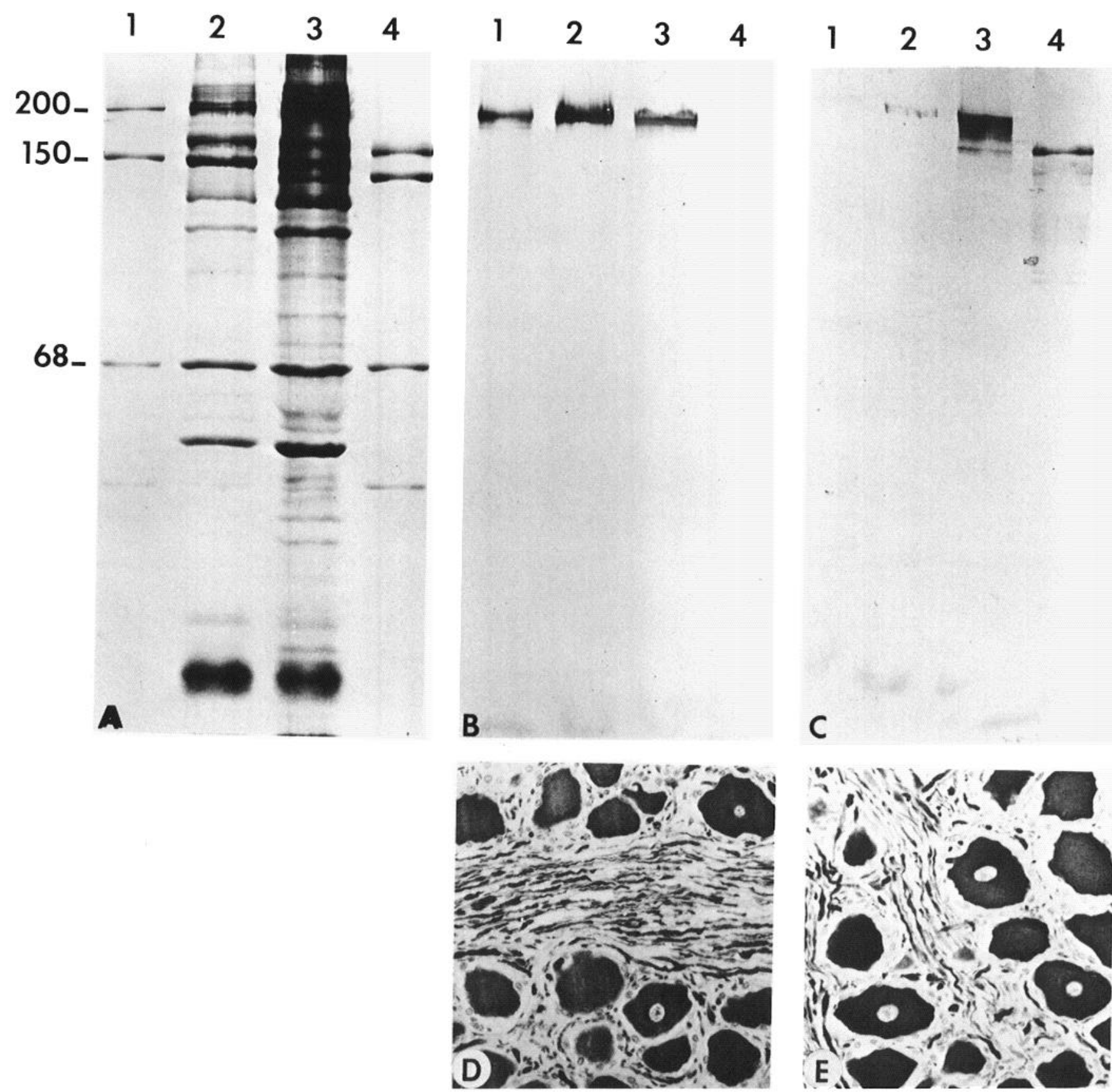

Figure 8. Immunochemical and immunohistochemical detection of nonphosphorylated or dephosphorylated NF200 in bovine trigeminal ganglion and nerve. A, A 7.5\% polyacrylamide gel stained with Coomassie blue, comparing the profile of proteins in the trigeminal nerve (lane 2) and ganglion (lane 3 ) with untreated (lane 1 ) and enzymatically dephosphorylated (lane 4) neurofilament extracts from spinal cord. Apparent $M$, values $\left(\times 10^{-3}\right)$ of the phosphorylated neurofilament polypeptides in lane $1(A)$ are indicated on the left. Gels identical to that in $A$ were used to generate nitrocellulose replicas that were incubated with Ta50 $(B)$ and $\mathrm{dP} 1(C)$. Ta50 and dP1 were also used to immunostain paraffin sections of trigeminal ganglion in $D$ and $E$, respectively. All magnifications $\times 400$.

is differentially phosphorylated in different regions of neurons. To probe this possibility further, we used PAP immunohistochemistry of bovine cerebellum, spinal cord, and trigeminal nerve and ganglion fixed in Bouin's medium, in combination with phosphatase treatment, to investigate whether the staining distribution of the $\mathrm{dP}$ series of monoclonal antibodies could be extended after tissue dephosphorylation (Fig. 7). In untreated tissue, dP1 intensely stained the perikarya, dendrites, and axons of nearly $100 \%$ of the Purkinje cells and of certain other cerebellar cortical neurons, while most other axons and dendrites were infrequently or weakly immunostained (Fig. 7A). However, following treatment with phosphatase, intense staining was seen in basket cell axon collaterals, as well as in many other axons and dendrites that had been completely unstained prior to phosphatase treatment (Fig. $7 B$ ). Using the same methodology, immunostaining patterns were compared, using a monoclonal antibody (Ta56) that recognizes an epitope on NF200 that is unchanged by dephosphorylation (Fig. 7, $C$ and $D$ ). Changes in the pattern of immunoreactivity for a phosphatedependent NF200 epitope were probed in the same manner, using Ta50 (Fig. 7, $E$ and $F$ ). In untreated tissue, Ta50 stained a group of structures similar to those recognized by $\mathrm{dP} 1$ after phosphatase treatment (compare Fig. 7, $E$ with $A$ ). After such treatment, however, much of the immunoreactivity was lost 
(Fig. $7 F$ ). In particular, there was a marked reduction in the staining of axons and dendrites. Treatment of tissues with trypsin before phosphatase did not enhance or extend the patterns obtained with phosphatase alone. Similar results were obtained with sections of spinal cord and trigeminal nerve and ganglion (see below).

\section{Identification of an endogenous form of a nonphosphorylated or dephosphorylated form of NF200 in trigeminal ganglion and nerve}

Immunostaining of Purkinje perikarya with the $\mathrm{dP}$ antibodies suggests the existence, in vivo, of a nonphosphorylated or dephosphorylated form of NF200, yet it was not detected in immunoblots of neurofilaments purified from white matter of bovine spinal cord. We interpreted these data as signifying that white-matter neurofilaments consist primarily of phosphorylated forms of NF200, since they are derived from an axonenriched source. Therefore, to demonstrate an endogenous, nonphosphorylated form of NF200, we considered it necessary to examine a tissue enriched in neuronal perikarya, i.e., trigeminal ganglion.

The Coomassie blue-stained gel in Figure $8 A$ shows the polypeptide composition of crude extracts from trigeminal nerve (lane 2) and trigeminal ganglion (lane 3) compared with phosphatase-treated (lane 4) and untreated (lane 1) neurofilaments from spinal cord. Tissue was homogenized in SDS, urea, and reducing agent to minimize any degradation of proteins and to maximize their extractability. Nitrocellulose replicas were prepared from gels identical to that in Figure 8 and were reacted with Ta50 (Fig. 4B) and dP1 (Fig. 4C). The phosphorylated form of NF200 in trigeminal nerve and ganglion is evident in the immunoblot stained with Ta50 in Figure $8 B$; this form of NF200 was equally distributed in both the nerve and ganglion homogenates. It migrates in an identical position to NF200 in untreated spinal cord neurofilaments. In contrast, the nonphosphorylated or dephosphorylated form of NF200 detected with $\mathrm{dP} 1$ was more abundant in the crude homogenate of trigeminal ganglion compared with a similar preparation from the trigeminal nerve (Fig. $8 \mathrm{C}$ ). Moreover, in trigeminal ganglion, $\mathrm{dP} 1$ stains a continuum of bands ranging in apparent molecular weight from 200,000 down to the position of dephosphorylated NF200 in the adjacent lane (lane 4). (Note that there is no immunoreactivity below this point.) Our interpretation of this pattern is that trigeminal ganglion contains various forms of NF200 ranging from nonphosphorylated forms through partially phosphorylated forms, since dP1 stains bands of increasing electrophoretic migration during progressive neurofilament dephosphorylation (see Carden et al., 1985). Bennett and DiLullo (1985) showed similar immunoreaction patterns for chicken NF150 as it becomes phosphorylated and demonstrated the simultancous presence of multiple phosphorylated forms of NF150 in cultured neurons.

The immunoperoxidase studies of trigeminal ganglion and nerve yielded results that corresponded to the immunochemical data. The monoclonal antibody to the phosphorylated form of NF200 (Ta50) stained trigeminal ganglion neurons and axons equally (Fig. $8 D$ ); following enzymatic dephosphorylation, the immunostaining of these neurons and axons was reduced, as shown for cerebellum (Fig. $7 F$ ). In contrast, dPl stained the perikarya of trigeminal ganglion neurons intensely (Fig. $8 E$ ), whereas immunoreactivity in axons diminished rapidly and progressively as they exited the ganglion to form branches of the trigeminal ncrve (data not shown). Enzymatic dephosphorylation of similar sections did not alter the staining of trigeminal ganglion neurons, but did increase the immunoreactivity seen in trigeminal nerves (data not shown) in a manner similar to that observed in cerebellum (Fig. $7 B$ ).

\section{Discussion}

The studies reported here describe for the first time a group of monoclonal antibodies (the $\mathrm{dP}$ series) that recognize exclusively the nonphosphorylated or enzymatically dephosphorylated form of NF200. These antibodies, therefore, recognize epitopes in NF200 that are phosphate-dependent, in that the presence of phosphate(s) at or near the antibody binding region changes the precise charge, structure, or conformation of the epitope and thereby its immunoreactivity. Phosphate-dependent epitopes in neurofilaments parallel the phenomenon observed with glycoproteins, in which the presence or absence of carbohydrate moieties dramatically changes antigenic properties (Alexander and Elder, 1984). The phosphate dependency of NF200 and NF150 epitopes has been described previously by Sternberger and Sternberger (1983). However, the new antibodies described here are the first generated by using enzymatically dephosphorylated neurofilament immunogens, and capable of recognizing only nonphosphorylated forms of phosphate-dependent epitopes in NF200.

It is important to note that previous anti-neurofilament monoclonal antibodies or antisera were generated by immunization with neurofilament proteins purified by conventional isolation techniques to obtain neurofilaments from nervous tissues rich in axons. In such preparations, NF200 and NF150 are highly phosphorylated (Carden et al., 1985; Geisler et al., 1985; Jones and Williams, 1982; Julien and Mushynski, 1982). Indeed, in our previous library of antibodies to neurofilament proteins (e.g., Carden et al., 1985; Lee et al., 1982b, 1984; Trojanowski et al., 1985) we obtained over 100 monoclonal antibodies that recognized phosphorylated forms of phosphate-dependent NF200 epitopes. All of these antibodies bind epitopes in the carboxy-terminal domain, the region containing the bulk of the phosphorylation sites (Geisler et al., 1985; Julien and Mushynski, 1983). Only one monoclonal antibody (Ta56) that recognized NF200 equally well before and after dephosphorylation was obtained. The epitope recognized by this antibody has, therefore, been termed phosphate-independent; it is located in the filament-binding core domain of NF200 (Carden et al., 1985).

If the trends suggested by our experience with antibodies gencratcd against NF200 represent a general phenomenon, then we may speculate that antisera raised to NF200 are composed largely of antibodies recognizing phosphate-dependent epitopes residing in the carboxy-terminal or peripheral domain (see also evidence in Carden et al., 1985). These antibodies would then require the presence of phosphate groups for much of their immunoreactivity. One of the most important implications of this statement is that antibodies raised to conventional preparations of NF200 might not be able to detect newly synthesized, and presumably nonphosphorylated, nascent forms of NF200this might explain limited immunostaining of neuronal perikarya with some antisera and monoclonal antibodies to neurofilament subunits (e.g., Dahl, 1983; Sternberger and Sternberger, 1983; Trojanowski et al., 1985).

It is important to emphasize that conventional procedures for the extraction of neurofilament proteins did not yield convincing immunochemical evidence for the presence of an endogenous form of nonphosphorylated or dephosphorylated NF200 despite the immunohistochemical evidence for its presence in cerebellum, spinal cord, and trigeminal ganglion. It was necessary to homogenize tissue using a Tris- $\mathrm{HCl}$ buffer containing SDS, urea, and beta-mercaptoethanol in order to identify this form of NF200 biochemically. Phosphorylated NF200 may be more resistant to spontaneous degradation than nascent, nonphosphorylated or incompletely phosphorylated NF200; the latter forms of NF200 may not survive prolonged extraction procedures. However, it is also important to select a region of the nervous system enriched in the nonphosphorylated form of NF200 in order to 
isolate this species of the largest neurofilament subunit. Thus, consistent with the immunohistochemical studies, the trigeminal ganglion was a better source for the immunochemical identification of this polypeptide than cerebellum or spinal cord.

The demonstration in previous studies (Sternberger and Sternberger, 1983; Trojanowski et al., 1985) that monoclonal antibodies recognizing phosphate-dependent NF200 epitopes stain certain perikarya as well as axons and dendrites shows that NF200 can be phosphorylated in all these neuronal regions. Similarly, reaction of the $\mathrm{dP}$ series of monoclonal antibodies with perikarya, dendrites, and axons of cerebellar Purkinje cells demonstrates the possibility that a nonphosphorylated NF200 epitope can exist in all parts of a neuron. Hence, phosphorylated and nonphosphorylated forms of NF200 may not necessarily be confined to mutually exclusive regions of all neurons. Indeed, a dynamic interchange of NF200 phosphorylation events should be considered as an explanation for part of the staining patterns obtained with the $\mathrm{dP}$ series of antibodies. These antibodies might be expected to recognize not only nascent NF200 but also forms once posttranslationally phosphorylated and subsequently dephosphorylated by the action of endogenous phosphatase.

Although the phosphorylation of many nervous system proteins has been extensively studied (Nestler and Greengard, 1984), there is little information on the role that phosphorylation plays in the metabolism and function of neurofilament proteins. The new monoclonal antibodies described here, combined with those in our previous library, should prove useful probes for investigating aspects of NF200 phosphorylation in vitro, both in developing and established neurons and in neurodegenerative disorders.

\section{References}

Alexander, S., and J. H. Elder (1984) Carbohydrate dramatically influences immune reactivity of antisera to viral glycoprotein antigens. Science 226: 1328-1330.

Bennett, G. S., and C. DiLullo (1985) Slow posttranslational modification of a neurofilament protein. J. Cell Biol. 100: 1799-1804.

Calvert, R., and B. H. Anderton (1982) In vivo metabolism of mammalian neurofilament polypeptides in developing and adult rat brain. FEBS Lett. 145: 171-175.

Carden, M. J., W. W. Schlaepfer, and V. M.-Y. Lee (1985) The structure, biochemical properties and immunogenicity of neurofilament peripheral regions are determined by phosphorylation. J. Biol. Chem. 260: 9805-9817.

Chin, T.-K., P. A. M. Eagles, and A. M. Maggs (1983) The proteolytic digestion of ox neurofilaments with trypsin and alpha-chymotrypsin. Biochem. J. 215: 239-252.

Czosnek, H., D. Soifer, and H. M. Wisniewski (1980) Studies on the biosynthesis of neurofilament proteins. J. Cell Biol. 85: 726-734.

Dahl, D. (1983) Immunohistochemical differences between neurofilaments in perikarya, dendrites and axons. Exp. Cell Res. 149: $397-$ 408.

Dahl, D., D. J. Selkoe, R. T. Pero, and A. Bignami (1982) Immunostaining of neurofibrillary tangles in Alzheimer's senile dementia with a neurofilament antisera. J. Neurosci. 2: 113-119.

Eagles, P. A. M., C. W. Bell, A. M. Maggs, C. Wais-Steider, and D. S. Gilbert (1978) Neurofilament structure and its enzymatic modification. In The Cytoskeletal and Contractile Networks of Non-muscle Cells, R. D. Goldman, ed., p. 57, Cold Spring Harbor Laboratories, Cold Spring Harbor, NY.

Gambetti, P., L. Autilio-Gambetti, G. Perry, G. Shecket, and R. C. Crane (1983) Antibodies to neurofibrillary tangles of Alzheimer's disease raised from human and animal neurofilament fractions. Lab. Invest. 49: 430-435.

Geisler, N., S. Fischer, J. Vanderkerckhove, J. Van Damme, U. Plessmann, and K. Weber (1985) Protein-chemical characterization of
NF-H, the largest mammalian neurofilament component; intermediate filament-type sequences followed by a unique carboxy-terminal extension. EMBO J. 4: 57-63.

Hirokawa, N., M. A. Glicksman, and M. B. Willard (1984) Organization of mammalian neurofilament polypeptides within the neuronal cytoskeleton. J. Cell Biol. 98: 1523-1536.

Hoftman, P. N., J. W. Griffin, and D. L. Price (1984) Control of axonal calibre by neurofilament transport. J. Cell Biol. 99: 705-714.

Jones, S. M., and R. C. Williams (1982) Phosphate content of mammalian neurofilaments. J. Biol. Chem. 257: 9902-9905.

Julien, J.-P., and W. E. Mushynski (1982) Multiple phosphorylation sites in mammalian neurofilament polypeptides. J. Biol. Chem. 257. $10467-10470$

Julien, J.-P., and W. E. Mushynski (1983) The distribution of phosphorylation sites among identified proteolytic fragments of mammalian neurofilaments. J. Biol. Chem. 258: 4019-4025.

Laemmli, U. K. (1970) Cleavage of structural proteins during the assembly of bacteriophage T4. Nature 227: 680-685.

Lazarides, E. (1982) Intermediate filaments. Annu. Rev. Biochem. 51: 219-245.

Lee, V. M.-Y. (in press) Neurofilament abnormalities in $\mathrm{PCl} 2$ cells: Comparison with neurofilament proteins of normal cultured rat sympathetic neurons. J. Neurosci.

Lee, V., M. Shelanski, and L. Greene (1981) Identification of neural and adrenal medullary surface membrane glycoproteins recognized by antisera to cultured rat sympathetic neurons and $\mathrm{PC} 12$ pheochromocytoma cells. Neuroscience 6: 2773-2786.

Lee, V. M.-Y., J. Q. Trojanowski, and W. W. Schlaepfer (1982a) Induction of neurofilament triplet proteins in PC1 2 cells by nerve growth factor. Brain Res. 238: 169-180.

Lee, V. M.-Y., H.-L. Wu, and W. W. Schlaepfer (1982b) Monoclonal antibodies recognize individual neurofilament triplet proteins. Proc. Natl. Acad. Sci. USA 79: 6089-6092.

Lee, V. M.-Y., C. D. Page, H.-L. Wu, and W. W. Schlaepfer (1984) Monoclonal antibodies to gel excised glial filament protein and their reactivity with other intermediate filament proteins. J. Neurochem. 42: 25-32.

Lewis, S. A., and N. J. Cowan (1985) Genetics, evolution, and expression of the 68,000 -mol-wt neurofilament protein: Isolation of a cloned cDNA probe. J. Cell Biol. 100: 843-850.

Nestler, E. J., and P. Greengard (1984) Protein Phosphorylation in the Nervous System, Wiley, New York.

O'Farrell, P. Z., H. M. Goodman, and P. H. O'Farrell (1977) High resolution two dimensional electrophoresis of basic as well as acidic proteins. Cell 12: 1133-1142.

Schlaepfer, W. W. (1984) Neurofilaments and the abnormal filaments of Alzheimer's disease. In Biological Aspects of Alzheimer's Disease, R. Katzman, ed., pp. 117-124, Banbury Report Series, Cold Spring Harbor Laboratories, Cold Spring Harbor, NY.

Schlaepfer, W. W., V. M.-Y Lee, and H.-L. Wu (1981) Assessment of immunological properties of neurofilament triplet proteins. Brain Res. 226: $259-272$.

Shecket, G., and R. J. Lasek (1978) Phosphorylation of $10 \mathrm{~nm}$ neurofilaments. In The Cytoskeletal and Contractile Networks of Nonmuscle Cells, R. D. Goldman, ed., p. 48, Cold Spring Harbor Laboratories, Cold Spring Harbor, NY.

Sternberger, L. A., and N. H. Sternberger (1983) Monoclonal antibodies distinguish phosphorylated and nonphosphorylated forms of neurofilaments in situ. Proc. Natl. Acad. Sci. USA 80: 6126-6130.

Trojanowski, J. Q., M. A. Obrocka, and V. M.-Y. Lee (1985) Distribution of bovine neurofilament subunits in neurons and neuronal processes: Immunohistochemical studies of bovine cerebellum with subunit specific monoclonal antibodies. J. Histochem. Cytochem. 33. $557-563$.

Weber, K., and N. Geisler (1984) Intermediate filaments-From wool alpha-keratins to neurofilaments: A structural overview. In Cancer Cells 1: The Transformed Phenotype, A. J. Levine, G. F. Van de Woude, W. C. Topp, and J. D. Watson, eds., pp. 153-159, Cold Spring Harbor Laboratories, Cold Spring Harbor, NY. 